\title{
Allocation of attention: Uncertainty effects when monitoring one or two visual gratings of noncontiguous spatial frequencies
}

\author{
ELIZABETH T. DAVIS \\ New York University, New York, New York 10003
}

\begin{abstract}
When an observer is visually presented with a sinusoidal grating, he will often do worse in detecting a given grating when he is uncertain about its spatial frequency than when he is certain. Theoretical explanations of such uncertainty effects assume that the observer has attentional control over multiple spatial-frequency channels. This attentional control can be selectively allocated. If one grating is presented on most of the trials, randomly intermixed with trials of gratings of other spatial frequencies, an experienced observer will use a stationary single-band attention strategy. If two gratings, separated in spatial frequency by four octaves, are randomly presented on most of the intermixed trials, an experienced observer will use a more complex attention strategy; he can monitor the spatial frequencies of the two extreme stimuli with little or no monitoring of intermediate spatial frequencies.
\end{abstract}

In everyday experience, human observers are often uncertain about the characteristics of complex visual scenes. By the methods of Fourier analysis, any complex scene can be decomposed into a set of sinusoidal gratings. Therefore, in studies of uncertainty effects, observers have been left uncertain about the spatial frequency of vertical sinusoidal gratings presented visually. Although the amount of uncertainty may have been quite small, the observers often did worse in detecting a given grating than they would have had they been certain of the spatial frequency. This reduced detectability is a spatialfrequency uncertainty effect, analogous to the frequency uncertainty effects reported in the auditory literature (e.g., Green \& Swets, 1966; Greenberg \& Larkin, 196:; MacMillan \& Schwartz, 1975; Swets \& Kristofferson, 1970). Spatial-frequency uncertainty effects have now been reported by a number of psychophysical investigators in vision (Cormack \& Blake, 1980; Davis, 1979; Davis \& Graham, 1981; Graham, Robson, \& Nachmias, 1978; Sekuler, Note 1).

Theoretical accounts of spatial-frequency uncertainty effects usually assume that the observer has attentional control over multiple sensory channels; each channel is maximally sensitive to a different spatial frequency and responds only to a restricted range of frequencies. (See Graham, 1980, for a review of the multiple spatial-frequency channels literature.)

The focus of this report is the degree to which an experienced observer can allocate attention in selectively monitoring the outputs of different spatial-

This research was partially supported by NSF Grant BNS-76. 18839 to Norma Graham and was conducted at Columbia University. I appreciate the helpful comments and suggestions of Norma Graham, Ted Adelson, Tony Movshon, and Ron Davis. frequency channels. First, can observers allocate more attention to the spatial frequency of the "primary" stimulus that is presented on a majority of the trials than to "secondary" stimuli presented randomly on the remainder of the trials? Second, can an experienced observer monitor the outputs of two noncontiguous sets of channels sensitive to very different spatial frequencies without monitoring the outputs of channels sensitive to intermediate spatial frequencies?

\section{METHOD}

\section{Experimental Conditions}

A "probe" technique similar to one used in audition by Greenberg and Larkin (1968) and by MacMillan and Schwartz (1975) was employed. There were two experimental conditions utilizing the "probe" technique, a one-primary condition and a two primaries condition.

In both conditions, there were "alone" sessions as well as "intermixed" sessions, each conducted on alternate days. In an alone session, stimuli of only orie spatial frequency were tested in a single block of trials and there was one block of trials for each spatial frequency used. In an "intermixed" session, primary and secondary stimuli were presented randomly within a block of trials, but only one stimulus was presented per trial.

For the one-primary condition, a primary stimulus with a spatial frequency of 4.0 cycles per degree (c/deg) was presented randomly on $95 \%$ of the intermixed trials for a total of 5,130 trials summed across the intermixed sessions. Secondary stimuli (probes) of spatial frequencies below the primary frequency (i.e., 1.0, 1.5, and $2.5 \mathrm{c} / \mathrm{deg}$ ) were presented randomly on the remaining $5 \%$ of the trials in every block of intermixed trials for one observer. For the other observer, however, the secondary stimuli were above the primary frequency (i.e., 6.5, 10.0, and $16.0 \mathrm{c} / \mathrm{deg}$ ). Each secondary stimulus was presented for a total of 90 trials across all intermixed sessions.

For the two-primaries condition, primary stimuli with spatial frequencies of 1.0 and $16.0 \mathrm{c} / \mathrm{deg}$ (4 octaves apart) were each presented randomly on $47.5 \%$ of the intermixed trials for a total of 2,565 trials across intermixed sessions. Secondary stimuli with intermediate spatial frequencies of $2.5,4.0$, and $6.5 \mathrm{c} / \mathrm{deg}$ were 
randomly presented on the remaining $5 \%$ of the intermixed trials for a total of 90 trials per secondary stimulus.

For both conditions, in the alone sessions each stimulus was presented, for a total of 360 trials summed across alone sessions in a given condition.

All trials were temporal two-interval forced-choice trials (without feedback) in which the observer had only to detect the presence of a stimulus. Each interval was demarked by a tone and had a duration of $100 \mathrm{msec}$ with abrupt onset and offset; the pause between intervals lasted approximately $200 \mathrm{msec}$. The observer initiated each trial by pushing a button.

On the basis of previous testing, each sinusoidal grating had been tested separately and its contrast had been set at approximately $90 \%$ correct response. (That is, each stimulus had the same apparent contrast, but not the same physical contrast.)

\begin{abstract}
Apparatus
The stimuli were vertical sinusoidal gratings produced by conventional Z-axis modulation (Campbell \& Green, 1965) on the face of a Tektronix $5103 \mathrm{~N}$ oscilloscope with a P-31 phosphor of green hue. An annular surround with an outer diameter of $31.75 \mathrm{~cm}$ and of approximately the same hue framed the oscilloscope. Both the scope and the surround had a mean luminance of $1.9 \mathrm{fL}$. All stimuli were viewed from a distance of $145 \mathrm{~cm}$ so that the vertical extent of the scope was $4 \mathrm{deg}$ of visual angle and the horizontal extent was $5.25 \mathrm{deg}$.

\section{Observers}

Two experienced observers participated in these experiments; one observer was the author. Both observers had had their vision corrected to normal acuity.
\end{abstract}

\section{RESULTS AND DISCUSSION}

The data were analyzed in terms of "difference" curves derived from the difference in sensitivity between "intermixed" and "alone" sessions for each stimulus. Sensitivity for the "intermixed" and "alone" sessions was measured in signal detection terms of $\mathrm{d}^{\prime}$ for two-interval forced choice trials as described in Green and Swets (1966, pp. 408-411).

Table 1 shows the $d^{\prime}$ values obtained for alone and intermixed sessions in the one-primary condition. Figure 1 shows the difference curve results. Notice that the uncertainty effect is smallest at the trough in the difference curve, corresponding to the primary frequency of $4.0 \mathrm{c} / \mathrm{deg}$. Conversely, the uncertainty

Table 1

Results of One-Primary Condition

\begin{tabular}{cccr}
\hline & \multicolumn{3}{c}{ Obtained d' Measures } \\
\cline { 2 - 4 } $\begin{array}{c}\text { Cycles } \\
\text { per Degree }\end{array}$ & $\begin{array}{c}\text { Alone } \\
\text { Sessions }\end{array}$ & $\begin{array}{c}\text { Intermixed } \\
\text { Sessions }\end{array}$ & $-\dot{\Delta d^{\prime}}$ \\
\hline & & Observer T.S. \\
1.0 & 1.93 & -.04 & 1.97 \\
1.5 & 2.16 & .74 & 1.42 \\
2.5 & 1.89 & 1.12 & .77 \\
4.0 & 2.05 & 1.66 & .39 \\
& & Observer E.D. & \\
4.0 & 1.76 & 1.41 & .35 \\
6.5 & 2.09 & 1.71 & .38 \\
10.0 & 1.86 & 1.14 & .72 \\
16.0 & 2.04 & .43 & 1.61 \\
\hline
\end{tabular}

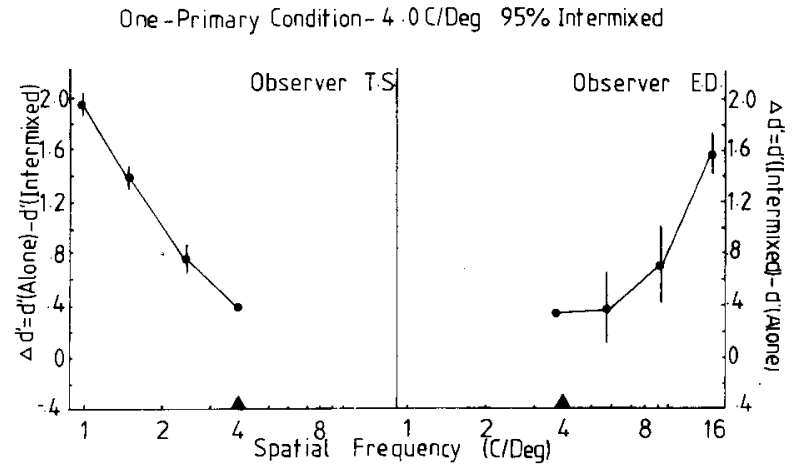

Figure 1. The one-primary difference curves obtained from two observers; observer T.S.'s data are shown on the left and observer E.D.'s data, on the right. The spatial frequency of the primary stimulus is $4.0 \mathrm{c} / \mathrm{deg}$ and is denoted by arrows on the horizontal axis; the primary was presented on $95 \%$ of the intermixed trials. The spatial frequency of the stimulus is plotted along the abscissa on a logarithmic scale. The difference in mean $d^{\prime}$ measures between alone and intermixed sessions is plotted on the ordinate. The vertical bars through the data points represent \pm 1 standard error.

effect is largest at the secondary frequency furthest from the $4.0-\mathrm{c} / \mathrm{deg}$ primary. Therefore, for both observers, the uncertainty effect tends to be "tuned" around the primary spatial frequency.

Observers are assumed to have attentional control over the multiple spatial-frequency channels because of the tuning found in the uncertainty effect for the one-primary condition. Attention strategies adapted from models in auditory psychophysics, as reported in Green and Swets (1966) and in Swets and Kristofferson (1970), may explain frequency uncertainty effects. The tuning of the uncertainty effects suggests that a single-band attention strategy was used when the different stimuli were intermixed from trial to trial. That is, the observer monitors a single spatial-frequency channel or a small subset of contiguous spatial-frequency channels sensitive to the 4.0-c/deg primary stimulus. ${ }^{1}$ (Notice that the distinction I am making between a single band and a single channel is that a single band may be composed of more than one contiguous spatial-frequency channel.) An uncertainty effect occurs because the observer is sometimes monitoring the "wrong" single band, one insensitive to the stimulus, and therefore does not detect the stimulus presented.

If the location of the single band remains stationary, the uncertainty effect should be sharply tuned, with only one trough in the difference curve. Furthermore, secondary stimuli with spatial frequencies outside the bandwidth of the monitored single band would be detected at chance level in the intermixed sessions. If the location of the single band is slowly switched from trial to trial, however, there should be little or possibly no tuning of the uncertainty effect. (Assume that the time course of switching takes longer than the duration of a $100-\mathrm{msec}$ stimulus in- 
Two-Primaries Condition - 1.0\& 16.0 ClDeg 95\% Intermixed

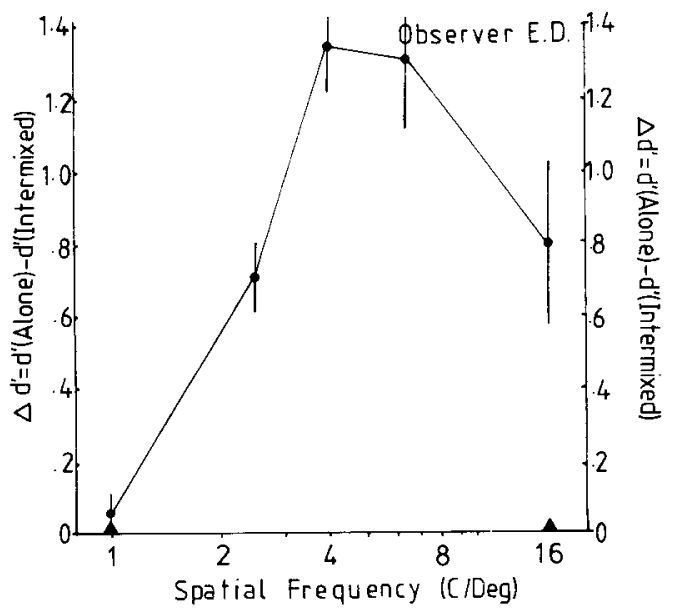

Figure 2. The two-primaries difference curve obtained from observer E.D. The spatial frequencies of the primaries were 1.0 and $16.0 \mathrm{c} / \mathrm{deg}$, each presented on $47.5 \%$ of the intermixed trials for a total of $95 \%$. The spatial frequencies of the two primary stimuli, each denoted by an arrow on the abscissa, were 4 octaves apart. Again, vertical bars through the data points represent \pm 1 standard error.

terval.) The sharp tuning of the uncertainty effect around the primary stimulus coupled with the chanceor near-chance-level detection of the secondary stimuli furthest from the $4.0-\mathrm{c} / \mathrm{deg}$ primary indicates that only one stationary single band was monitored in the one-primary condition.

To determine how an observer monitors noncontiguous spatial-frequency channels, let's now examine the results of the two-primaries condition shown in Figure 2. There is no significant uncertainty effect at the lower primary frequency $(1.0 \mathrm{c} / \mathrm{deg})$ and a small uncertainty effect at the higher primary frequency $(16.0 \mathrm{c} / \mathrm{deg})$. The uncertainty effects are largest for the intermediate secondary frequencies; in fact, detection of the $4.0-$ and $6.5-\mathrm{c} / \mathrm{deg}$ gratings in the intermixed sessions is near chance level. Thus, when two primary stimuli are 4 octaves apart in spatial frequency, the tuning in the uncertainty effect is centered around both primary frequencies.
Likely models of attention strategies to explain the two-primaries' results would be the slow-switching single-band model or the attention-sharing-over-twostationary-single-bands model, or some combination of these two models; each single band would be centered at the spatial frequency of a primary stimulus. ${ }^{2}$

To analyze the two-primaries' results more quantitatively, consider only the data of the two primary stimuli. The upper portion of Table 2 shows the obtained $d^{\prime}$ measures for each primary stimulus in the alone and intermixed sessions. The $d^{\prime}$ measure for the alone sessions was used to predict $d^{\prime}$ estimates for the intermixed sessions according to switching or attention-sharing models.

For the pure slow-switching single-band model, assume that at a given moment the observer monitors only one single band, that his attention can be switched between two single bands (each sensitive to one of the primary frequencies) without monitoring stimuli of intermediate frequency, and that it takes him longer than the duration of a 100 -msec stimulus interval to switch attention between the two single bands. The prediction of the slow-switching single-band model for intermixed trials in which the $1.0-\mathrm{c} / \mathrm{deg}$ primary is presented are as follows:

$$
P_{1 \text { Int }}=p\left(P_{1 \text { Alone }}\right)+(1-p)(.5),
$$

where $p$ is the percentage of intermixed trials on which the observer monitors the single band sensitive to the $1.0-\mathrm{c} / \mathrm{deg}$ primary. $\mathrm{P}_{1 \text { Alone }}$ and $\mathrm{P}_{11 \mathrm{nt}}$ are the percent correct in detecting the $1.0-\mathrm{c} / \mathrm{deg}$ primary within alone and intermixed blocks of trials, respectively. On trials in which the observer does not attend the $1.0-\mathrm{c} / \mathrm{deg}$ primary (i.e., $1-\mathrm{p}$ ), he correctly guesses the stimulus interval on half of the trials. The predictions for the $16.0-\mathrm{c} / \mathrm{deg}$ primary are based on a similar equation as follows:

$$
P_{16 \text { Int }}=(1-p) P_{16 \text { Alone }}+p(.5),
$$

where $P_{16 \text { Alone }}$ and $P_{16 \text { Int }}$ are the percent correct in detecting the $16.0-\mathrm{c} / \mathrm{deg}$ primary within alone and intermixed blocks of trials, respectively. Table 2 shows the predicted $d^{\prime}$ estimates for the intermixed sessions based on the slow-switching single-band

Table 2

\begin{tabular}{|c|c|c|c|c|c|c|c|c|}
\hline \multirow[b]{3}{*}{$\begin{array}{c}\text { Cycles } \\
\text { per Degree }\end{array}$} & & & & & \multicolumn{4}{|c|}{ Predicted $d^{\prime}$ Measures for Intermixed Sessions } \\
\hline & \multicolumn{2}{|c|}{$\begin{array}{c}\text { Obtained } \mathrm{d}^{\prime} \\
\text { Measures }\end{array}$} & \multicolumn{2}{|c|}{$\begin{array}{c}\text { Actual Percent } \\
\text { Correct Measures }\end{array}$} & \multicolumn{2}{|c|}{$\begin{array}{l}\text { Slow-Switching Single- } \\
\text { Band Model }\end{array}$} & \multicolumn{2}{|c|}{$\begin{array}{l}\text { Attention Sharing Over Two } \\
\text { Stationary Single-Bands Mode }\end{array}$} \\
\hline & $\begin{array}{l}\text { Alone } \\
\text { Sessions }\end{array}$ & $\begin{array}{l}\text { Intermixed } \\
\text { Sessions }\end{array}$ & $\begin{array}{l}\text { Alone } \\
\text { Sessions }\end{array}$ & $\begin{array}{c}\text { Intermixed } \\
\text { Sessions }\end{array}$ & $\begin{array}{c}p= \\
29.95\end{array}$ & $\begin{array}{c}p= \\
97.70\end{array}$ & $\begin{array}{l}\text { Maximum Out- } \\
\text { put Rule }\end{array}$ & $\begin{array}{c}\text { Adding of } \\
\text { Outputs Rule }\end{array}$ \\
\hline $\begin{array}{r}1.0 \\
16.0\end{array}$ & $\begin{array}{l}1.43 \\
1.97\end{array}$ & $\begin{array}{l}1.38 \\
1.17\end{array}$ & $\begin{array}{l}84.5 \\
92.0\end{array}$ & $\begin{array}{l}83.7 \\
79.6\end{array}$ & $\begin{array}{r}.36 \\
1.17\end{array}$ & $\begin{array}{r}1.38 \\
.04\end{array}$ & $\begin{array}{l}1.155 \\
1.740\end{array}$ & $\begin{array}{l}1.01 \\
1.40\end{array}$ \\
\hline
\end{tabular}

Analyses of Two-Primaries Condition 
model (see Elliot, cited in Swets, 1964, Appendix I); to obtain the correct $d^{\prime}$ estimate for one primary, the predicted $d^{\prime}$ estimate for the other primary is much too low. Thus, we can reject this pure version of the slow-switching single-band model. [Note that with a fast-switching single-band model in which attention switching is much faster than $100 \mathrm{msec}$ (e.g., Kristofferson, 1967) and both single bands are equally monitored within a stimulus interval, the predictions for switching will be indistinguishable from the attention-sharing model described below.]

For the pure attention-sharing model, assume that the information from the two single bands is utilized according to either a maximum output rule or an adding of outputs rule. The maximum output rule states that the observer monitors the output of each single band in both temporal intervals of a twointerval forced-choice trial and selects the interval in which the biggest response occurred in one of the monitored single bands. That is, the observer examines $m=2 n$ outputs, where $n$ is the number of monitored single bands (two in this case) and there are two outputs for each single band (one for each interval), for a total of $m$ outputs. The probability of correctly reporting the stimulus interval for stimulus " $i$ " in an intermixed session is as follows:

$$
P_{i}=P_{i m}+\left(1-P_{i m}\right)[(n-1) /(m-1)],
$$

where $P_{i m}$ is the probability that the relevant single band for stimulus " $i$ " produced the largest output in the stimulus interval (viz, Creelman, 1960). The probability that the irrelevant single band produced the largest response in the stimulus interval is $\left(1-\mathrm{P}_{\mathrm{im}}\right)$ $[(n-1) /(m-1)]$; in this case, the observer still chooses the correct interval. In Table 2 , the $P_{i}$ values have been converted to the predicted intermixed $d^{\prime}$ values according to the maximum output rule.

The adding-of-outputs rule states that within each temporal interval the outputs of the two single bands are added together and the observer chooses the interval with the largest combined output. The probability of correctly reporting the stimulus interval for stimulus " $i$ " in an intermixed session is as follows:

$$
\hat{\mathrm{d}}_{\mathrm{i}}^{\prime}=\mathrm{d}_{\mathrm{i}}^{\prime} / \sqrt{\mathrm{M}},
$$

where $\mathrm{d}^{\prime}{ }_{\mathrm{i}}$ is the sensitivity measure obtained for stimulus " $i$ " in the alone sessions (i.e., when the observer is certain of the spatial frequency) and $M$ is the number of monitored single bands (viz, Creelman, 1960). (The $d^{\prime}$ measures for the other $M-1$ single bands are assumed to have means of zero.)

The maximum output rule predicts somewhat smaller uncertainty effects than does the adding-ofoutputs rule. In either case, both rules predict too large an uncertainty effect for the $1.0-\mathrm{c} / \mathrm{deg}$ primary, although the maximum output rule is more accurate. Furthermore, both rules predict too small an uncer- tainty effect for the $16.0-\mathrm{c} / \mathrm{deg}$ primary, although the adding-of-outputs rule is more accurate.

The attention strategy the observer uses may be a combination of the two models considered-on some trials, only the $1.0-\mathrm{c} / \mathrm{deg}$ primary was attended, and on other trials, both the $1.0-$ and $16.0-\mathrm{c} / \mathrm{deg}$ primaries were attended. According to the maximum output rule, the observer must monitor both primary stimuli on $76.5 \%$ of the intermixed trials in order to obtain a d' measure of 1.16 for the $16.0-\mathrm{c} / \mathrm{deg}$ primary in the intermixed sessions. For the $1.0-\mathrm{c} / \mathrm{deg}$ primary, the predicted $\mathrm{d}^{\prime}$ measure is 1.24 in the intermixed sessions, which is slightly lower than the obtained d' measure of 1.38 . According to the addingof-outputs rule, the observer must monitor both primary stimuli on $84 \%$ of the intermixed trials in order to obtain a $\mathrm{d}^{\prime}$ measure of 1.17 for the $16.0-\mathrm{c} / \mathrm{deg}$ primary in the intermixed sessions. For the $1.0-\mathrm{c} / \mathrm{deg}$ primary, the predicted $\mathrm{d}^{\prime}$ measure is 1.08 in the intermixed sessions, which is significantly lower than the obtained $\mathrm{d}^{\prime}$ measure of 1.38 . Thus, the predictions of the maximum output rule in the combination model of attention are closer to the values obtained in the two-primaries condition.

Results of the two-primaries condition indicate that an experienced observer can maximize his detection of two visual gratings of widely separated spatial frequencies by allocating attention over two noncontiguous sets of spatial-frequency channels with little or no monitoring of the intermediate channels. The observer does this by using a rather complex combined attention strategy-switching attention between one stationary and two stationary single bands, with each single band centered on the spatial frequency of a primary stimulus.

\section{CONCLUSIONS}

In summary, several conclusions can be drawn from these data. First, the spatial-frequency uncertainty effects probably depend upon attentional control over multiple mechanisms, such as multiple spatial-frequency channels. Second, observers tended to use the most efficient attention strategy. If only one primary was presented on most of the intermixed trials, a stationary single-band attention strategy was used. If two primaries were presented on most of the intermixed trials, however, a more complex attention strategy was used; attention was switched between simultaneously monitoring one or two stationary single bands, each centered on one of the primary spatial frequencies. Finally, an experienced observer could monitor two noncontiguous sets of spatial-frequency channels with little or no monitoring of the intermediate channels. The observer thereby reduced the amount of the uncertainty effect for the two primary stimuli being attended, but detection of most secondary stimuli of intermediate spatial frequencies was near chance. 


\section{REFERENCE NOTE}

1. Sekuler, R. Stimulus uncertainty: $A$ link between the laboratory and the real world. Paper presented at the Tenth Symposium of the Center for Visual Science, University of Rochester, June 1977.

\section{REFERENCES}

Balt, K., \& Sekuler, R. Models of stimulus uncertainty in motion perception. Psychological Review, 1980, 87, 435-469.

Campeell, F., \& Green, D. Optical and retinal factors affecting visual resolution. Journal of Physiology, 1965, 181, 576-593.

Cormack, R., \& Blake, R. Do the two eyes constitute separate visual channels? Science, 1980, 207, 1100-1101.

Creelman, C. Detection of signals of uncertain frequency. Journal of the Acoustical Society of America, 1960, 32, 805-810.

Davis, E. Spatial frequency uncertainty effects in the detection of visual sinusoidal gratings. Unpublished doctoral dissertation, Columbia University, New York, 1979.

Davis, E., \& GrahaM, N. Spatial frequency uncertainty effects in the detection of sinusoidal gratings. Vision Research, 1981, 21, 705-712.

Graham, N. Psychophysics of spatial-frequency channels. In M. Kubovy \& J. Pomerantz (Eds.), Perceptual organization. Hillsdale, N.J: Erlbaum, 1980.

Graham, N., Robson, J., \& Nachmias, J. Grating summation in fovea and periphery. Vision Research, 1978, 18, 815-826.

Green, D. M., \& SwE'T, J. A. Signal detection theory and psychophysics. New York: Wiley, 1966.

Greenberg, G. S., \& Larkin, W. D. Frequency-response characteristics of auditory observers detecting signals of a single frequency in noise: The probe-signal method. Journal of the Acoustical Society of America, 1968, 44, 1513-1523.

Kristofferson, A. B. Attention and psychophysical time. Acta Psychologica, 1967, 27, 93-100.

MacMillan, N., \& Schwartz, M. A probe-signal investigation of uncertain-frequency detection. Journal of the Acoustical Society of America, 1975, 58, 1051-1058.

SwETS, J. A. Signal detection and recognition by human observers. New York: Wiley, 1964.

Swets, J. A., \& Kristofferson, A. B. Attention. Annual Review of Psychology, 1970, 21, 339-366.

\section{NOTES}

1. At threshold, the output of each channel is assumed to be independently perturbed by noise. If there were inhibition between channels, however, this could increase the magnitude of the uncertainty effect. For instance, suppose the channel(s) sensitive to the 4.0-c/deg grating inhibited (i.e., reduced the output of channel(s) sensitive to the $16.0-\mathrm{c} / \mathrm{deg}$ grating, and vice versa. Also, suppose that the larger the output of one channel, the greater its inhibition of other channels. If the observer attended only the $4.0-\mathrm{c} / \mathrm{deg}$ grating when the $16.0-\mathrm{c} / \mathrm{deg}$ grating was presented, the output of the monitored single band (the one sensitive to the $4.0-\mathrm{c} / \mathrm{deg}$ grating) would be lower during the stimulus interval than during the blank interval. Thus, the observer would report that the stimulus had occurred during the blank interval and would be wrong. In this case, in the intermixed sessions it would even be possible to obtain negative d' values significantly below chance. However, no intermixed $d^{\prime}$ values were significantly below zero in these experiments.

2. The midway model that Ball and Sekuler (1980) have recently proposed is not consistent with the data from the two-primaries condition; their model would have predicted the smallest uncertainty effect for stimuli of intermediate spatial frequency. The results of the two-primaries condition, however, do not cast doubts on the validity of the midway model, because the two primaries are 4 octaves apart in spatial frequency and no single band used had a bandwidth so wide that it would be sensitive to both primary frequencies; in such a case, the midway model is not relevant.

(Received for publication July 21,1980 ; revision accepted February 9, 1981.) 